\title{
Ginzburg-Landau theory of the zigzag transition in quasi-one-dimensional classical Wigner crystals
}

\author{
J. E. Galván-Moya and F. M. Peeters* \\ Department of Physics, University of Antwerp, Groenenborgerlaan 171, B-2020, Antwerpen, Belgium
}

(Received 24 June 2011; published 18 October 2011)

\begin{abstract}
We present a mean-field description of the zigzag phase transition of a quasi-one-dimensional system of strongly interacting particles, with interaction potential $r^{-n} e^{-r / \lambda}$, that are confined by a power-law potential $\left(y^{\alpha}\right)$. The parameters of the resulting one-dimensional Ginzburg-Landau theory are determined analytically for different values of $\alpha$ and $n$. Close to the transition point for the zigzag phase transition, the scaling behavior of the order parameter is determined. For $\alpha=2$, the zigzag transition from a single to a double chain is of second order, while for $\alpha>2$, the one-chain configuration is always unstable and, for $\alpha<2$, the one-chain ordered state becomes unstable at a certain critical density, resulting in jumps of single particles out of the chain.
\end{abstract}

DOI: 10.1103/PhysRevB.84.134106

PACS number(s): 05.20.-y, 61.50.-f, 63.20.-e, 37.10.Ty

\section{INTRODUCTION}

During the last two decades, the interest in self-organized systems has increased enormously both experimentally and theoretically due to its importance in solid-state physics and plasma physics, as well as in atomic physics. Wigner crystals are an elementary example of self-organization, which has been realized in very diverse systems as, e.g., electrons on liquid helium ${ }^{1}$ by using the well-known Paul and Penning traps ${ }^{2,3}$ to confine ions in a limited region, in dusty plasma, ${ }^{4}$ and more recently using static and radio-frequency electromagnetic potentials where crystallization was realized through laser cooling. ${ }^{5}$ Additionally, it has been proposed that these structures can be used for a possible implementation of a scalable quantum information processor ${ }^{6,7}$ and as quasi-onedimensional (Q1D) Wigner crystals. ${ }^{8}$ The theoretical analysis of these crystal structures has been realized previously for three-dimensional (3D),,${ }^{9,10}$ two-dimensional (2D), ${ }^{11-13}$ and Q1D (Refs. 14 and 15) systems. From those studies, it was shown that structural phase transitions can be induced by varying the strength of the external confinement potential and/or the density of particles.

In this paper, we concentrate on the ordered state of identical particles that are confined in a Q1D channel. When the particles move in $2 \mathrm{D}$ and are confined by a parabolic ${ }^{14}$ or hard-wall ${ }^{16}$ potential in one of the in-plane directions, the particles arrange themselves in parallel chains at low temperature. Previously, it was found ${ }^{14}$ that, with increasing density (or decreasing strength of the confinement potential), the system passes through a sequence of first- and secondorder phase transitions, where at each point the number of chains changes. Of particular interest to us is the oneto two-chain transition, which for a parabolic confinement potential was found to be a second-order phase transition that occurs as a zigzag transition. Such a transition was observed experimentally ${ }^{17-20}$ in systems with a finite number of particles, and the effects of a narrow channel and finite size of the system on the diffusion were recently analyzed in Refs. 21 and 22. Recently, it was found theoretically ${ }^{23}$ that the analytic form of the confinement potential is very important for the occurrence of the zigzag transition and the order of the phase transition. Therefore, in this paper, we generalize the previous analysis to an arbitrary power-law confinement potential (i.e., $y^{\alpha}$ ) and also to arbitrary interparticle interaction, which we model by $r^{-n} e^{-r / \lambda}$, which simulates most of the relevant experimental particle-particle interactions. With this model potential, we can simulate both short- and long-range interactions.

We are interested in the behavior of the system at the zigzag transition, i.e., at the critical point. This can be viewed as a spontaneous symmetry breaking and we will cast the problem into a mean-field theory based on Landau's theory of phase transitions. In this way, we will construct a Ginzburg-Landau theory for the single- to two-chain transition in a quasi-one-dimensional system of interacting particles. We generalize the approach of Refs. 15 and 24 to arbitrary power-law confinement and interparticle interaction potential. We obtain a Ginzburg-Landau equation for the order parameter close to the transition point, and determine all the relevant parameters in this equation. The order parameter is the distance of the particles from the trap axis. By considering a large number of particles and using the local density approximation, we can consider the crystal as a continuum, so that the order parameter becomes a field.

This paper is organized as follows. In Sec. II, we describe the model system and, using Landau theory, we find the behavior of the system close to the transition point. Next, we derive a Ginzburg-Landau equation for the system finding the dispersion relation. In Sec. III, the results for the critical point and the normal mode spectrum are discussed. Our conclusions and a discussion of possible quantum effects are given in Sec. IV.

\section{THEORETICAL FRAMEWORK}

We consider a two-dimensional system consisting of $N$ particles with mass $m$ and charge $q$, which are allowed to move in the $x-y$ plane. The charged particles interact through a repulsive interaction potential; they are free to move in the $x$ direction, but are confined by a one-dimensional potential, which limits their motion in the $y$ direction. The total energy of the system is given by

$$
E=\frac{1}{2} m \sum_{i=1}^{N} \dot{\mathbf{r}}_{i}^{2}+V_{\text {conf }}+V_{\text {int }}
$$


where $V_{\text {conf }}$ and $V_{\text {int }}$ are the confinement and interaction potential respectively, given by

$$
\begin{aligned}
V_{\text {conf }} & =\frac{1}{2} m v_{t}^{2} R^{2} \sum_{i=1}^{N} \frac{\left|y_{i}\right|^{\alpha}}{R^{\alpha}}, \\
V_{\text {int }} & =\sum_{i=1}^{N} \sum_{j>i}^{N} V_{\text {pair }}\left(r_{i j}\right),
\end{aligned}
$$

where $V_{\text {pair }}\left(r_{i j}\right)$ represents the interparticle interaction. The latter one will be taken as a screened power-law potential as follows:

$$
V_{\text {pair }}=\frac{q^{2}}{\epsilon R} \frac{R^{n} e^{-r_{i j} / \lambda}}{r_{i j}^{n}},
$$

where $r_{i j}=\left|\mathbf{r}_{i}-\mathbf{r}_{j}\right|$ represents the relative position between the $i$ th and the $j$ th particles, the exponent $n$ is an integer, and $\epsilon$ is the dielectric constant of the medium the particles are moving in. In the above, $R$ is an arbitrary length parameter, which we introduced to guarantee the right units. The energy can be written in dimensionless form

$$
E=\sum_{i=1}^{\infty} \dot{\mathbf{r}}_{i}^{2}+v^{2} \sum_{i=1}^{N}\left|y_{i}\right|^{\alpha}+\sum_{i=1}^{N} \sum_{j>i}^{N} \frac{e^{-\kappa r_{i j}}}{r_{i j}^{n}}
$$

with dimensionless frequency $v$ given by $v=v_{t} / \omega_{0}$, while $\omega_{0}$ measures the strength of the confinement potential and $t_{0}=1 / \omega_{0}$ is the unit of time. The energy is expressed in units of $E_{0}=\left(m \omega_{0}^{2} / 2\right)^{n /(n+\alpha)}\left(q^{2} / \epsilon\right)^{\alpha /(n+\alpha)} R^{(2 n-\alpha) /(n+\alpha)}$ and all distances are expressed in units of $r_{0}=\left(2 q^{2} /\right.$ $\left.m \omega_{0}^{2} \epsilon\right)^{1 /(n+\alpha)} R^{(n+\alpha-3) /(n+\alpha)}$. Additionally, the dimensionless parameter $\kappa=r_{0} / \lambda$ represents the screening parameter of the potential. Limiting cases of this interaction potential are as follows: Yukawa potential $(n=1)$, power-law potential $(\kappa=$ $0)$, Coulomb potential $(\kappa=0, n=1)$, and dipole interaction $(\kappa=0, n=3)$. We introduce a dimensionless linear density $\eta$ defined as the number of particles per unit of length along the unconfined direction.

In Ref. 23, it was demonstrated that, for $\alpha>2$, the onechain configuration is not stable for any values of $\eta$ and $v$. Only for $\alpha=2$ does the system exhibit a continuous transition from the one- to the two-chain configuration (i.e., zigzag transition) at a transition point defined by a critical density $\left(\eta_{c}\right)$ or a critical frequency $\left(v_{c}\right)$. For $\alpha<2$, the ground-state configuration of the particles is, below $\eta_{c}$ or above $v_{c}$, arranged in a single chain. Beyond this critical point, particles are expelled one by one from this chain to positions parallel to the chain.

For the special case of $\alpha=2$ and before the transition point, the particles crystallize around the minimum point of the confinement potential $V$, at the positions $\mathbf{r}_{i}^{\text {linear }}=(i / \eta) \mathbf{e}_{\mathbf{x}}$ with $i$ an integer. The stability of the linear chain along the $x$ axis requires a relative transverse trap frequency exceeding a threshold value $v_{c}$ or a linear density smaller than $\eta_{c}$. At this critical point, the configuration has a structural instability, such that for $v<v_{c}$ or $\eta>\eta_{c}$, the particles are organized in a zigzag structure, ordered in two chains with equilibrium positions $\mathbf{r}_{i}^{\text {zigzag }}=(i / \eta) \mathbf{e}_{\mathbf{x}}+(-1)^{i}(c / \eta) \mathbf{e}_{\mathbf{y}}$, where $c$ is a real and positive constant, $d=c / \eta$ represents the distance of each particle from the confinement potential minimum, and $D=2 d$ indicates the lateral separation between the two chains.

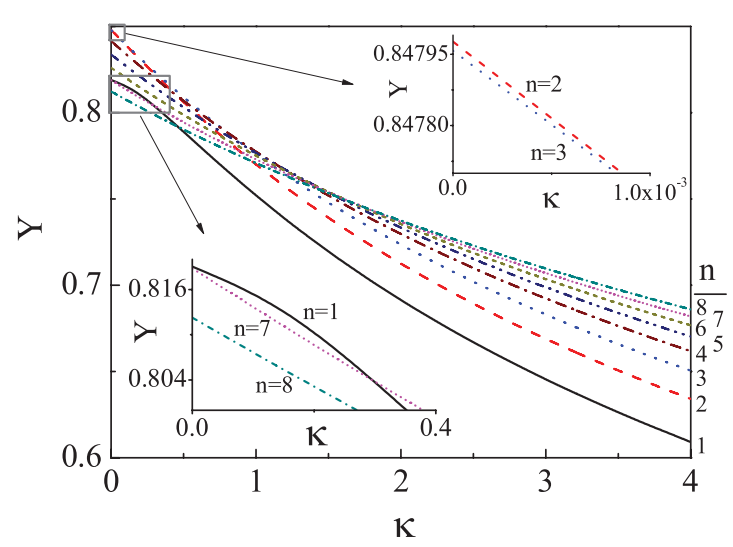

FIG. 1. (Color online) The proportionality coefficient $Y(v ; n, \kappa)$ as a function of $\kappa$ for different values of $n$ with $v=1$.

\section{A. Landau theory for the zigzag transition}

For the case $\alpha=2$ and near the zigzag regime, we follow the Landau theory approach of Ref. 23, and expand the total potential energy of the system as a function of the order parameter $c$ in a polynomial, i.e., $V(c)=V_{1 c h}-A c^{2}+B c^{4}$, where $V_{1 c h}$ represents the potential energy for the one-chain configuration. By minimizing the potential energy, we obtain the condition

$$
2 \sum_{j=0}^{\infty}\left[n \eta^{n}+\kappa \eta^{n-1}(2 j+1)\right] \frac{e^{-\kappa \frac{2 j+1}{\eta}}}{(2 j+1)^{n+2}}-\frac{v^{2}}{\eta^{2}}=0 .
$$

From this equation, we obtain the value of $\eta_{c}(v)$ or $v_{c}(\eta)$ at which the single-chain configuration becomes unstable. Considering $\eta_{c}$ and expanding the potential energy around this critical value, we find that the order parameter close to the transition point is given by

$$
c=Y(v: n, \kappa)\left|\eta-\eta_{c}\right|^{\frac{1}{2}},
$$

with $Y(v ; n, \kappa)=\sqrt{Y_{B} Y_{C}-Y_{A} Y_{D}} / Y_{B}$, where $Y_{A}=n \eta_{c}^{n} S_{2}+$ $\eta_{c}^{n-1} \kappa S_{1}-v^{2} / 2 \eta_{c}^{2}, \quad Y_{B}=2 n(n+2) \eta_{c}^{n} S_{4}+2(2 n+1) \eta_{c}^{n-1} \kappa$ $S_{3}+2 \eta_{c}^{n-2} \kappa^{2} S_{2}, \quad Y_{C}=n^{2} \eta_{c}^{n-1} S_{2}+(2 n-1) \eta_{c}^{n-2} \kappa S_{1}+\eta_{c}^{n-3}$ $\kappa^{2} S_{0}+v^{2} / \eta_{c}^{3}, \quad Y_{D}=2 n^{2}(n+2) \eta_{c}^{n-1} S_{4}+2[n(3 n+1)-1]$ $\eta_{c}^{n-2} \kappa S_{3}+2(3 n-1) \eta_{c}^{n-3} \kappa^{2} S_{2}+2 \eta_{c}^{n-4} \kappa^{3} S_{1}$, with

$$
S_{k}=\sum_{i=0}^{\infty} \frac{e^{-\kappa \frac{2 i+1}{\eta_{c}}}}{(2 i+1)^{n+k}}
$$

The value of $Y=Y(v ; n, \kappa)$ is plotted in Fig. 1 as a function of $\kappa$ for different values of $n$ and a fixed value $v=1$. Notice that the curves $Y(n, \kappa)$ for different values of $n$ cross each other at some value of $\kappa$. The critical exponent of the order parameter of the zigzag transition [Eq. (6)] was verified experimentally on a low-dimensional dusty plasma in Ref. 25 and theoretically in Refs. 23, 15, 26, and 27.

\section{B. Ginzburg-Landau Lagrangian for the zigzag phase transition}

Recently, this zigzag transition (for $\alpha=2, \kappa=0$, and $n=$ 1) was cast into a mean-field description resulting in similar expressions as in the Landau theory of phase transitions. This resulted in a one-dimensional Ginzburg-Landau-type nonlinear field theory. ${ }^{24}$ Here, we will extend the previous calculation to the more general problem described by the 
energy Eq. (4). We start by considering the system in the situation that the one-chain configuration is stable but that it is close to the transition point. The equilibrium positions of all the particles are along the $x$ axis. We consider small oscillations around the equilibrium position of each particle as follows: $x_{i}^{\text {lin }}=(i / \eta)+x_{i}$ and $y_{i}^{\text {lin }}=0+y_{i}$. Then, the relative position between the particles can be written as

$$
r_{i j}=\left[1+\frac{\tau_{i j}+\epsilon_{i j}}{A_{i j}}\right]^{1 / 2}\left(A_{i j}\right)^{1 / 2},
$$

with $A_{i j}=\left(\Delta_{i j}\right)^{2}, \tau_{i j}=2 \Delta_{i j}\left(x_{i}-x_{j}\right)$, and $\epsilon_{i j}=\left(x_{i}-x_{j}\right)^{2}$ $+\left(y_{i}-y_{j}\right)^{2}$, where $\Delta_{i j}=(i-j) / \eta$. Now, we assume that the vibration amplitudes in the axial and transverse directions are much smaller than the distance between the particles, i.e., $\tau_{i j}, \epsilon_{i j} \ll A_{i j}$. We expand Eq. (8) and the exponential term as

$$
\begin{aligned}
r_{i j} \approx & \Delta_{i j}+\left(x_{i}-x_{j}\right) \\
& +\left(1-\frac{\left(x_{i}-x_{j}\right)}{\Delta_{i j}}+\frac{\left(x_{i}-x_{j}\right)^{2}}{\left(\Delta_{i j}\right)^{2}}\right) \frac{\left(y_{i}-y_{j}\right)^{2}}{2 \Delta_{i j}}
\end{aligned}
$$

$$
\begin{aligned}
e^{-\kappa r_{i j}} \approx & e^{-\kappa \Delta_{i j}}\left[1-\kappa\left(x_{i}-x_{j}\right)+\kappa^{2} \frac{\left(x_{i}-x_{j}\right)^{2}}{2}\right. \\
& \left.-\kappa \frac{\left(y_{i}-y_{j}\right)^{2}}{2 \Delta_{i j}}\right]
\end{aligned}
$$

and similar to the $n$th power of the inverse of Eq. (8), i.e., $1 / r_{i j}^{n}$, as a Newton binomial around the equilibrium positions. These expansions result in a decomposition of the total potential as

$$
V=v^{2} \sum_{i=1}^{\infty}\left|y_{i}\right|^{\alpha}+\sum_{l}^{\infty} V_{\text {int }}^{(l)},
$$

where the label $l$ indicates the order of the expansion. Each order of the expansion of the interaction potential can be written as

$$
V_{\text {int }}^{(l)}=\frac{1}{2} \sum_{i \neq j}^{\infty} W_{i j}^{(l)},
$$

where the expansion terms up to fourth order are given by

$$
\begin{gathered}
W_{i j}^{(0)}=\frac{e^{-\kappa \Delta_{i j}}}{\Delta_{i j}^{n}}, \\
W_{i j}^{(1)}=-\frac{e^{-\kappa \Delta_{i j}}}{\left(\Delta_{i j}\right)^{n+1}}\left[\vartheta_{i j} n+\kappa \Delta_{i j}\right]\left(x_{i}-x_{j}\right), \\
W_{i j}^{(2)}=\frac{e^{-\kappa \Delta_{i j}}}{2\left(\Delta_{i j}\right)^{n+2}}\left\{\left[n(n+1)-2 \vartheta_{i j} n \kappa \Delta_{i j}+\kappa^{2} \Delta_{i j}^{2}\right]\left(x_{i}-x_{j}\right)^{2}-\left(n+\kappa \Delta_{i j}\right)\left(y_{i}-y_{j}\right)^{2}\right\} \\
W_{i j}^{(3)}=\frac{e^{-\kappa \Delta_{i j}}}{2\left(\Delta_{i j}\right)^{n+3}} n\left(x_{i}-x_{j}\right)\left[-\left(\frac{\vartheta_{i j}}{3}\left(n^{2}+3 n+2\right)+(n+1) \kappa \Delta_{i j}+\vartheta_{i j} \kappa^{2} \Delta_{i j}^{2}\right)\left(x_{i}-x_{j}\right)^{2}\right. \\
\left.+\left[\vartheta_{i j}(n+2)+\kappa \Delta_{i j}\right]\left(y_{i}-y_{j}\right)^{2}\right] \\
W_{i j}^{(4)}=\frac{e^{-\kappa \Delta_{i j}}}{2\left(\Delta_{i j}\right)^{n+4} n}\left[\left(\frac{1}{12}\left(n^{3}+6 n^{2}+11 n+6\right)+3\left(n^{2}+3 n+2\right) \kappa \Delta_{i j}+2(n+1) \kappa^{2} \Delta_{i j}^{2}\right)\left(x_{i}-x_{j}\right)^{4}\right. \\
-\left(\frac{1}{2}\left(n^{2}+5 n+6\right)+\left[9(n+2) \vartheta_{i j}+2(n+1)\right] \kappa \Delta_{i j}+2 \kappa^{2} \Delta_{i j}^{2}\right)\left(x_{i}-x_{j}\right)^{2}\left(y_{i}-y_{j}\right)^{2} \\
\left.+\left(\frac{1}{4}(n+2)+2 \kappa \Delta_{i j}\right)\left(y_{i}-y_{j}\right)^{4}\right]
\end{gathered}
$$

with $\vartheta_{i j}=\Delta_{i j} /\left|\Delta_{i j}\right|$. It is sufficient to restrict ourselves to terms up to the fourth order and, thus, the potential can be written as $V_{\text {int }} \approx V_{\text {int }}^{(0)}+V_{\text {int }}^{(1)}+V_{\text {int }}^{(2)}+V_{\text {int }}^{(3)}+V_{\text {int }}^{(4)}$.

\section{Representation in reciprocal space}

Now, we assume that the particles are pinned in the longitudinal direction and that they can only oscillate in the transverse direction $\left(x_{i}=0\right)$. Therefore, their normal axial modes can be neglected and we can discard the coupling to the longitudinal modes. In this regime, we find that $W_{i j}^{(0)}=e^{-\kappa \Delta_{i j}} /\left(\Delta_{i j}^{n}\right), \quad W_{i j}^{(1)}=0, \quad W_{i j}^{(2)}=-\left(n+\kappa \Delta_{i j}\right)\left(y_{i}-\right.$ $\left.y_{j}\right)^{2} e^{-\kappa \Delta_{i j}} /\left[2\left(\Delta_{i j}\right)^{n+2}\right], W_{i j}^{(3)}=0, \quad$ and $\quad W_{i j}^{(4)}=n[(n+2)+$ $\left.8 \kappa \Delta_{i j}\right]\left(y_{i}-y_{j}\right)^{4} e^{-\kappa \Delta_{i j}} /\left[8\left(\Delta_{i j}\right)^{n+4}\right]$.
In order to find the representation in reciprocal space, we define the normal modes of vibration in the transversal direction with wave vector $k$ as $\Psi_{k}=\psi_{k}^{(+)}-i \psi_{k}^{(-)}$with amplitude $\left|\psi_{k}\right|^{2}=\psi_{k}^{(+) 2}+\psi_{k}^{(-) 2}$. Following the standard process to find this representation as shown in, e.g., Ref. 15 and using Plancherel's theorem ${ }^{28}$ for the confinement potential transformation, the different terms of the potential become

$$
\begin{gathered}
V_{\text {conf }}=\frac{v^{2}}{\sqrt{N}} \sum_{k>0}\left|\psi_{k}\right|^{\alpha}, \\
V_{\text {int }}^{(1)}=0, \\
V_{\text {int }}^{(2)}=\sum_{k>0} \omega_{\perp}(n, \kappa, k)^{2} \psi_{k}^{2},
\end{gathered}
$$




$$
\begin{gathered}
V_{\text {int }}^{(3)}=0, \\
V_{\text {int }}^{(4)}=\sum_{k_{1}+k_{2}+k_{3}+k_{4}=0} A\left(k_{1}, k_{2}, k_{3}, k_{4}\right) \prod_{m=1}^{4} \psi_{k_{m}},
\end{gathered}
$$

where $\quad \omega_{\perp}(n, \kappa, k)^{2}=-\omega(n, \tilde{\kappa}, k)^{2}, \quad A\left(k_{1}, k_{2}, k_{3}, k_{4}\right)=$ $n \eta^{n+4}\left[(n+2) A_{0}\left(k_{1}, k_{2}, k_{3}, k_{4}\right)+8 \tilde{\kappa} A_{1}\left(k_{1}, k_{2}, k_{3}, k_{4}\right)\right] / 8$,

with $\tilde{\kappa}=\kappa / \eta$ and

$$
\begin{aligned}
\omega(n, \tilde{\kappa}, k)^{2}= & 2 \eta^{n+2}\left[n \sum_{j=1}^{\infty} \frac{e^{-j \tilde{\kappa}}}{j^{n+2}} \sin ^{2}\left(j \frac{k}{2}\right)\right. \\
& \left.+\kappa \sum_{j=1}^{\infty} \frac{e^{-j \tilde{\kappa}}}{j^{n+1}} \sin ^{2}\left(j \frac{k}{2}\right)\right], \\
A_{0}\left(k_{1}, k_{2}, k_{3}, k_{4}\right) & =\frac{2}{N} \sum_{j>0} \frac{e^{-j \tilde{\kappa}}}{j^{n+4}} \prod_{m=1}^{4} \sin \left(j \frac{k_{m}}{2}\right), \\
A_{1}\left(k_{1}, k_{2}, k_{3}, k_{4}\right) & =\frac{2}{N} \sum_{j>0} \frac{e^{-j \tilde{\kappa}}}{j^{n+3}} \prod_{m=1}^{4} \sin \left(j \frac{k_{m}}{2}\right) .
\end{aligned}
$$

Due to the condition that the motion of the particles is restricted to the longitudinal direction, it becomes apparent that the first- and third-order terms of the interaction potential will be zero, and additionally we know that the first derivative equals zero because it is the necessary condition to have an equilibrium configuration.

\section{Minimum frequency of the interaction potential}

From the definition of $\omega_{\perp}(n, \kappa, k)^{2}$, we find that its minimum value is located at $k_{0}=\pi$. Let us expand for $k$ around this value $\left(k=k_{0}-\delta k\right)$, and we obtain

$$
\begin{aligned}
\omega_{\perp}\left(n, \kappa, k_{0}-\delta k\right)^{2}= & \omega_{\perp}\left(n, \tilde{\kappa}, k_{0}\right)^{2} \\
& +h(n, \tilde{\kappa})^{2} \delta k^{2}+O\left(\delta k^{4}\right), \\
A\left(k_{1}, k_{2}, k_{3}, k_{4}\right)= & \frac{1}{2 N} \mathcal{A}(n, \tilde{\kappa})+O\left(\delta k^{2}\right),
\end{aligned}
$$

where $\omega_{\perp}\left(n, \tilde{\kappa}, k_{0}\right)^{2}=-\varpi(n, \tilde{\kappa})^{2}$ with

$$
\begin{aligned}
\varpi(n, \tilde{\kappa})^{2}= & \left(\frac{\eta}{2}\right)^{n+2} e^{-\tilde{\kappa}}\left[2 n \Phi\left(e^{-2 \tilde{\kappa}}, n+2, \frac{1}{2}\right)\right. \\
& \left.+4 \tilde{\kappa} \Phi\left(e^{-2 \tilde{\kappa}}, n+1, \frac{1}{2}\right)\right] \\
h(n, \tilde{\kappa})^{2}= & \left(\frac{\eta}{2}\right)^{n} \sum_{j=1}^{2}(-1)^{j+1} e^{-j \tilde{\kappa}}\left[\frac{n}{2} \Phi\left(e^{-2 \tilde{\kappa}}, n, \frac{j}{2}\right)\right. \\
& \left.+\tilde{\kappa} \Phi\left(e^{-2 \tilde{\kappa}}, n-1, \frac{j}{2}\right)\right]
\end{aligned}
$$

$$
\begin{aligned}
\mathcal{A}(n, \tilde{\kappa})= & \left(\frac{\eta}{2}\right)^{n+4} n e^{-\tilde{\kappa}}\left[\left(\frac{n}{2}+1\right) \Phi\left(e^{-2 \tilde{\kappa}}, n+4, \frac{1}{2}\right)\right. \\
& \left.+8 \tilde{\kappa} \Phi\left(e^{-2 \tilde{\kappa}}, n+3, \frac{1}{2}\right)\right],
\end{aligned}
$$

where $\Phi(z, s, a)$ is the Lerch transcendent defined as $\Phi(z, s, a)=\sum_{k=0}^{\infty} z^{k} /(k+a)^{s}$. In Table I, we show the limiting behavior of these terms. It is important to note that the square of the transverse frequency is negative.

\section{Stability of the system}

The system is stable when the second-order term of the total potential energy (i.e., the coefficient of $\psi_{k}^{2}$ ) is minimum. For the parabolic case $(\alpha=2)$, the confinement potential term contributes to the second order of the total potential energy, then

$$
V^{(2)}=\sum_{k>0} \omega_{\perp}(n, \kappa, k)^{2} \psi_{k}^{2},
$$

where the transverse frequency is

$$
\omega_{\perp}(n, \kappa, k)^{2}=v^{2}-\omega(n, \tilde{\kappa}, k)^{2}
$$

and we note that its minimum value is reached for $k_{0}=\pi$. Thus, the critical value for the confinement frequency is given by

$$
v_{c}^{2}(n, \kappa)=\varpi(n, \tilde{\kappa})^{2} .
$$

When $v>v_{c}$, the ground-state configuration is a one-chain organization of the particles. For $v<v_{c}$, the linear chain is unstable and the particles are arranged in a two-chain structure through a zigzag organization. When $v$ is sufficiently close to the critical value $v_{c}$, an effective potential can be derived for the transverse normal modes $\psi_{k}$ with wave vector $\tilde{k}=k_{0}-$ $\delta k$, such that $\delta k \ll 1$. The second-order term of the effective potential is given by Eq. (17), where its coefficient, Eq. (15a), can now be written as

$$
\omega_{\perp}\left(n, \kappa, k_{0}-\delta k\right)^{2}=\delta_{v}(n, \tilde{\kappa})+h(n, \tilde{\kappa})^{2} \delta k^{2},
$$

where $\delta_{v}(n, \tilde{\kappa})=v^{2}-v_{c}(n, \tilde{\kappa})^{2}$. In the limiting case of a Coulomb interparticle potential $(\kappa=0, n=1)$ and considering $\eta=1$, we find $v_{c}(1,0)=\sqrt{7 \zeta(3)} / 2=1.45038$, $h(1,0)=\sqrt{\log (2) / 2}=0.58871$, and $\mathcal{A}(1,0)=93 \zeta(5) / 64=$ 1.50679 , which agrees with the results of Refs. 24 and 15. In Table II, we show the values of these terms for different interaction potentials. Notice that the critical confinement frequency $v_{c}$ decreasing with increasing screening $\kappa$, and it increases with increasing density. The relation between $v_{c}$

TABLE I. Behavior of the coefficients in the Ginzburg-Landau equation in two limiting cases, where $\tilde{\kappa}=\kappa / \eta$.

\begin{tabular}{lcc}
\hline \hline & $\tilde{\kappa} \ll 1$ & $\tilde{\kappa} \gg 1$ \\
\hline$\varpi(n, \tilde{\kappa})^{2}$ & $2 n\left(\frac{\eta}{2}\right)^{n+2} e^{-\tilde{\kappa}} \sum_{j=0}^{\infty} \frac{1-2 j \tilde{\kappa}}{(j+1 / 2)^{n+2}}$ & $2 n \eta^{n+2} \tilde{\kappa} e^{-\tilde{\kappa}}$ \\
$h(n, \tilde{\kappa})^{2}$ & $\frac{n}{2}\left(\frac{\eta}{2}\right)^{n} \sum_{j=0}^{\infty}(1-2 j \tilde{\kappa})\left(\frac{1}{(j+1 / 2)^{n}}-\frac{1}{(j+1)^{n}}\right)$ & $\left(\frac{\eta}{2}\right)^{n} \tilde{\kappa} e^{-\tilde{\kappa}} \sum_{j=0}^{\infty} \frac{e^{-2 j \tilde{\kappa}}}{(j+1 / 2)^{n-1}}$ \\
$\mathcal{A}(n, \tilde{\kappa})$ & $n\left(\frac{n}{2}+1\right)\left(\frac{\eta}{2}\right)^{n+4} \sum_{j=0}^{\infty} \frac{1-2 j \tilde{\kappa}}{(j+1 / 2)^{n+4}}$ & $8 n\left(\frac{\eta}{2}\right)^{n+4} \tilde{\kappa} e^{-\tilde{\kappa}} \sum_{j=0}^{\infty} \frac{e^{-2 j \tilde{\kappa}}}{(j+1 / 2)^{n+4}}$ \\
\hline \hline
\end{tabular}


TABLE II. Values of the critical parameters for different values of $n$ and $\kappa$.

\begin{tabular}{|c|c|c|c|c|c|c|c|c|c|c|}
\hline$n$ & $\kappa$ & $v_{c}(n, \tilde{\kappa})$ & $\begin{array}{c}\eta=0.50 \\
h(n, \tilde{\kappa})\end{array}$ & $\mathcal{A}(n, \tilde{\kappa})$ & $v_{c}(n, \tilde{\kappa})$ & $\begin{array}{c}\eta=1.00 \\
h(n, \tilde{\kappa})\end{array}$ & $\mathcal{A}(n, \tilde{\kappa})$ & $v_{c}(n, \tilde{\kappa})$ & $\begin{array}{l}\eta=1.50 \\
h(n, \tilde{\kappa})\end{array}$ & $\mathcal{A}(n, \tilde{\kappa})$ \\
\hline 1 & 0.5 & 0.43113 & 0.38151 & 0.06332 & 1.36621 & 0.57569 & 2.13008 & 4.02285 & 0.82789 & 62.6067 \\
\hline 1 & 1.0 & 0.31885 & 0.30221 & 0.04019 & 1.21941 & 0.53954 & 2.02615 & 3.86422 & 0.81415 & 68.1625 \\
\hline 1 & 2.0 & 0.15131 & 0.15008 & 0.01002 & 0.90183 & 0.42740 & 1.28595 & 3.44901 & 0.76302 & 64.8368 \\
\hline 2 & 0.5 & 0.37195 & 0.35188 & 0.06901 & 1.74736 & 0.80730 & 4.85728 & 7.52638 & 1.71598 & 299.492 \\
\hline 2 & 1.0 & 0.26019 & 0.25394 & 0.04230 & 1.48781 & 0.70376 & 4.41649 & 6.98946 & 1.61460 & 310.865 \\
\hline 2 & 2.0 & 0.11720 & 0.11676 & 0.01030 & 1.04076 & 0.50788 & 2.70688 & 5.95124 & 1.40752 & 282.655 \\
\hline 3 & 0.5 & 0.30339 & 0.29542 & 0.05605 & 2.06259 & 0.99201 & 8.19081 & 12.7470 & 3.04303 & 1047.17 \\
\hline 3 & 1.0 & 0.20566 & 0.20332 & 0.03331 & 1.71625 & 0.83557 & 7.17465 & 11.6677 & 2.80582 & 1048.42 \\
\hline 3 & 2.0 & 0.08952 & 0.08936 & 0.00794 & 1.16342 & 0.57508 & 4.26315 & 9.70860 & 2.36334 & 918.354 \\
\hline
\end{tabular}

and $n$ depends on the density value, as will be discussed later.

Additionally, from a simple expansion of the dispersion relation (18) around the equilibrium positions and for values of the frequency and density close to their critical values, the value of the parameter $c$ can be found from the nonlinear algebraic equation

$$
\begin{aligned}
& v^{2}-2 \eta^{n+2} {\left[n \sum_{j=1}^{\infty} \frac{e^{-\tilde{\kappa} \sqrt{(2 j-1)^{2}+c^{2}}}}{\left[(2 j-1)^{2}+c^{2}\right]^{\frac{n+2}{2}}}\right.} \\
&\left.+\tilde{\kappa} \sum_{j=1}^{\infty} \frac{e^{-\tilde{\kappa} \sqrt{(2 j-1)^{2}+c^{2}}}}{\left[(2 j-1)^{2}+c^{2}\right]^{\frac{n+1}{2}}}\right]=0 .
\end{aligned}
$$

\section{Continuum approximation}

Close to the transition point $(\delta k \ll 1)$, the transverse deviation of the particles is very small and we can use a continuum approach for these modes. In doing so, we replace the discrete sum over $\tilde{k}$ by an integral $\sum_{k} \rightarrow \int d(\delta k) N / 2 \pi$. Using the Fourier transform, we obtain a continuous form for the modes $\psi(x)$ as follows: $\psi_{k}=\int d x \psi(x) e^{-i \delta k x} / \sqrt{N}$. Then, the remaining terms of the potential become

$$
V^{(\alpha)}=\frac{1}{2} \int v^{2}|\psi(x)|^{\alpha} d x,
$$

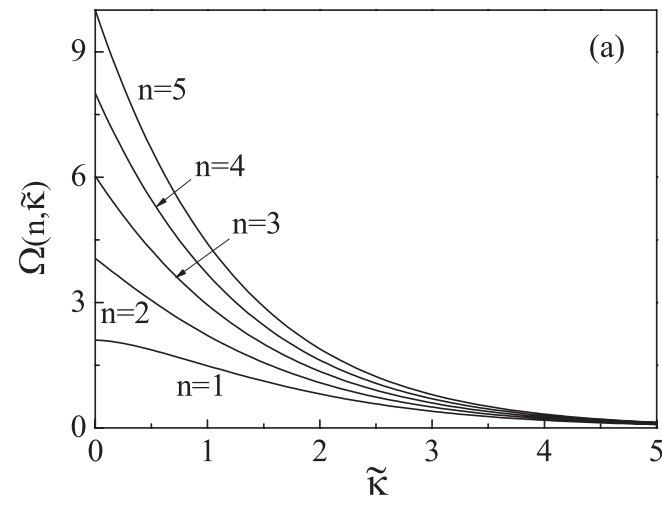

$$
\begin{aligned}
V^{(2)} & =\frac{1}{2} \int\left[-\varpi(n, \tilde{\kappa})^{2} \psi(x)^{2}+h(n, \tilde{\kappa})^{2}\left(\frac{\partial \psi}{\partial x}\right)^{2}\right] d x \\
V^{(4)} & =\frac{1}{2} \int \mathcal{A}(n, \tilde{\kappa}) \psi(x)^{4} d x .
\end{aligned}
$$

Finally, we obtain the Lagrangian $L=\int \mathcal{L}(x) d x$, where the Lagrangian density $\mathcal{L}(x)$ reads as

$$
\begin{aligned}
\mathcal{L}(x)= & \frac{1}{2}\left\{\left[\partial_{t} \psi(x)\right]^{2}-h(n, \tilde{\kappa})^{2}\left[\partial_{x} \psi(x)\right]^{2}+\varpi(n, \tilde{\kappa})^{2} \psi(x)^{2}\right. \\
& \left.-v^{2}|\psi(x)|^{\alpha}-\mathcal{A}(n, \tilde{\kappa}) \psi(x)^{4}\right\} .
\end{aligned}
$$

In the special case $\alpha=2, \kappa=0, n=1$, this Lagrangian density is the one found in Ref. 24, and it has the form of a Ginzburg-Landau equation (Refs. 24 and 29). Defining $\varphi(x)=\eta \psi(x)$ and $\tilde{v}(n, \tilde{\kappa})^{2}=v(n, \tilde{\kappa})^{2} / \eta^{n+\alpha}$, we may find from Eq. (23) an expression for the potential energy density $\mathcal{V}$ :

$$
\frac{2 \mathcal{V}}{\eta^{n}}=K(n, \tilde{\kappa}) \varphi(x)^{4}+\tilde{v}(n, \tilde{\kappa})^{2}|\varphi(x)|^{\alpha}-\Omega(n, \tilde{\kappa}) \varphi(x)^{2},
$$

with the real positive coefficients $\Omega(n, \tilde{\kappa})=\varpi(n, \tilde{\kappa})^{2} / \eta^{n+2}$ and $K(n, \tilde{\kappa})=\mathcal{A}(n, \tilde{\kappa}) / \eta^{n+4}$, which are plotted in Fig. 2 as a function of $\tilde{\kappa}$ for different values of $n$. Notice that both coefficients are positive and decrease with increasing $\tilde{\kappa}$. Now, the density $\eta$ plays the role of a scaling parameter in

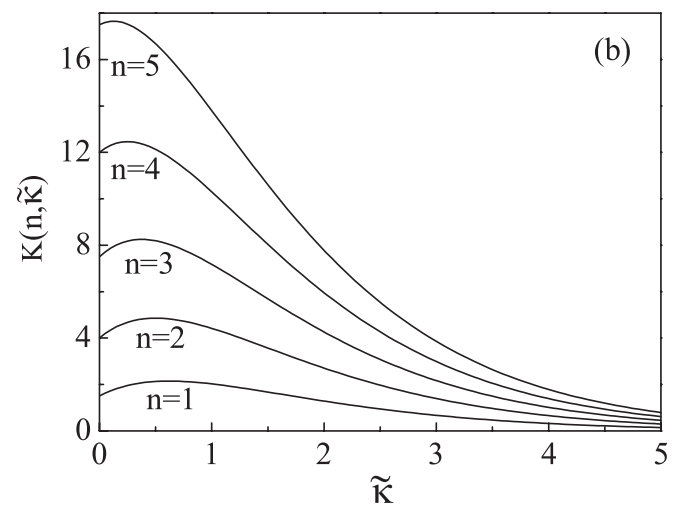

FIG. 2. Ginzburg-Landau coefficients for the potential energy density [Eq. (24)] as a function of the screening parameter $\tilde{\kappa}=\kappa / \eta$ for different values of $n$. 


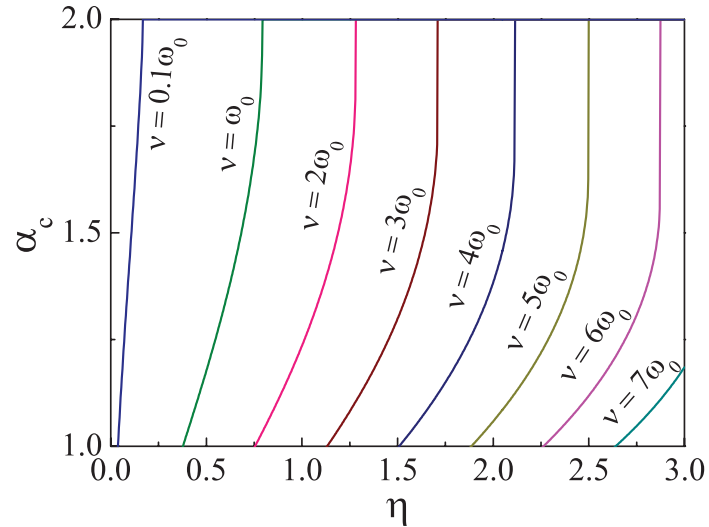

FIG. 3. (Color online) Critical value of the exponent of the confinement frequency as a function of the density for different values of the strength of the confinement potential. We took the parameters $n=1$ and $\kappa=1$.

the potential energy density $(\mathcal{V})$, in the screening parameter $(\kappa)$, in the strength of the confinement $(v)$, and in the order parameter $(\psi)$. For $\alpha=2$, we find the usual Landau energy expression for a second-order phase transition

$$
\frac{2 \mathcal{V}}{\eta^{n}}=K(n, \tilde{\kappa}) \varphi(x)^{4}+\left[\tilde{v}(n, \tilde{\kappa})^{2}-\Omega(n, \tilde{\kappa})\right] \varphi(x)^{2} .
$$

\section{Equation of motion}

From Eq. (23), we obtain the equation of motion for $\psi(x)$ as follows:

$$
\begin{aligned}
& \partial_{t}^{2} \psi(x)-h(n, \tilde{\kappa})^{2} \partial_{x}^{2} \psi(x)-\varpi(n, \tilde{\kappa})^{2} \psi(x) \\
& \quad+\frac{\alpha v^{2}}{2} \operatorname{sign}[\psi(x)]|\psi(x)|^{\alpha-1}+2 \mathcal{A}(n, \tilde{\kappa}) \psi(x)^{3}=0 .
\end{aligned}
$$

In this context, the order parameter $\psi(x)$ represents a continuous version for the value of $c$, which is the distance of the particles $(d=c / \eta)$ from the minimum of the confinement potential. When the order parameter varies slowly in space, the time-independent version of Eq. (26) becomes

$$
\varpi(n, \tilde{\kappa})^{2} \psi-\frac{\alpha v^{2}}{2} \operatorname{sign}(\psi)|\psi|^{\alpha-1}-2 \mathcal{A}(n, \tilde{\kappa}) \psi^{3}=0 .
$$

We note that, for $\alpha<1$, a one-chain configuration is not allowed because $\psi=0$ is not a solution of Eq. (27) in this case.

Considering $\alpha \geqslant 1$ and defining $\tilde{a}(n, \tilde{\kappa})=\alpha v^{2} / 2 \eta^{n+2}$ $\Omega(n, \tilde{\kappa})$ and $\tilde{b}(n, \tilde{\kappa})=2 \eta^{2} K(n, \tilde{\kappa}) / \Omega(n, \tilde{\kappa})$, the latter equation is reduced to

$$
1-\tilde{a}(n, \tilde{\kappa})|\psi|^{\alpha-2}-\tilde{b}(n, \tilde{\kappa}) \psi^{2}=0 .
$$

TABLE III. Order parameter for different values of $\alpha$.

\begin{tabular}{cc}
\hline \hline$\alpha$ & $\psi$ \\
\hline 2 & $\sqrt{(1-\tilde{a}) / \tilde{b}}$ \\
3 & $\left(-\tilde{a}+\sqrt{\tilde{a}^{2}+4 \tilde{b}}\right) / 2 \tilde{b}$ \\
4 & $1 / \sqrt{\tilde{a}+\tilde{b}}$ \\
\hline
\end{tabular}

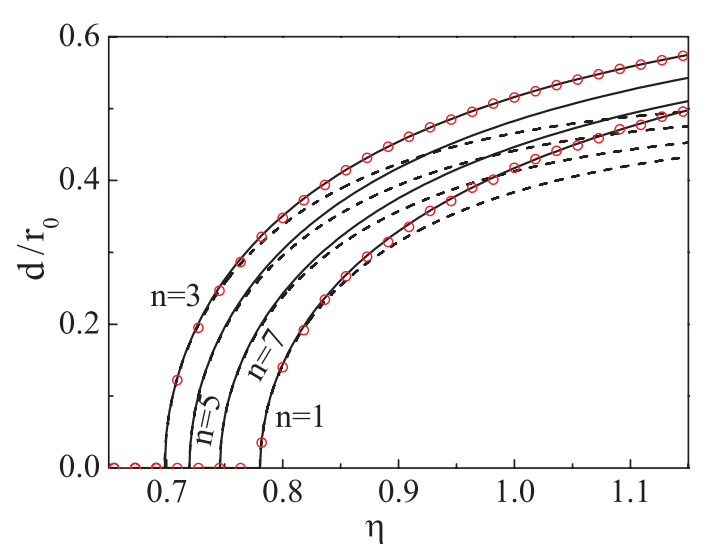

FIG. 4. (Color online) Displacement from the $x$ axis as a function of the linear density for $v=1$. The dashed lines are obtained from the Landau theory, while the solid lines are the solutions of Eq. (21). The open circles represent the results of our Monte Carlo simulations for $n=1$ and 3 .

For $\alpha=2$, this equation results in a second-order transition, from the single-chain (i.e., $\psi=0$ ) to the zigzag (i.e., $\psi \neq 0$ ) configuration, with the critical point defined by $\tilde{a}(n, \tilde{\kappa})=1$, which in fact is a generalization of Eq. (21).

For $1<\alpha<2$ and minimizing Eq. (28), we find

$$
2 \Omega(n, \tilde{\kappa})-\left(\frac{\alpha_{c}^{2} v^{2}}{2 \eta^{n+\alpha_{c}}}\right)^{\frac{2}{4-\alpha_{c}}}\left(\frac{4 \alpha_{c}}{2-\alpha_{c}} K(n, \tilde{\kappa})\right)^{\frac{2-\alpha_{c}}{4-\alpha_{c}}}=0,
$$

which represents a nonlinear equation for the critical exponent of the confinement potential $\left(\alpha_{c}\right)$, which is the minimum value of $\alpha$ for which a one-chain configuration is the ground-state configuration. From Eq. (29), we note that this critical value will be at most equal to 2 , as shown in Fig. 3 for different values of the strength of the confinement frequency.

Finally, we may find analytical expressions for the order parameter from Eq. (28) for different values of $\alpha \geqslant 2$, which are given in Table III. Notice that it is always possible to find a $\psi \neq 0$, which indicates that the single-chain configuration is always unstable when $\alpha>2$. For $\alpha=2$, we find $\psi \neq 0$ only when $\tilde{a}(n, \tilde{\kappa})<1$.

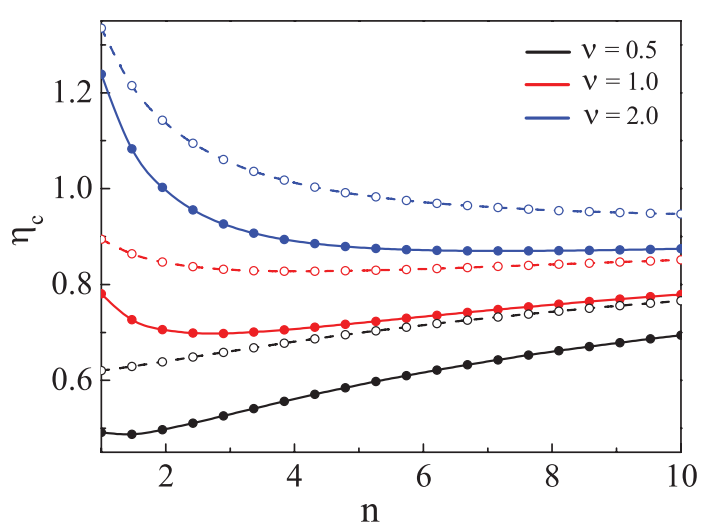

FIG. 5. (Color online) Critical linear density as a function of the $n$ exponent of the interparticle interaction for different values of the parabolic confinement frequency. The (solid and dashed) lines are the prediction of the Landau theory and the (solid and open) circles are found with the present method (for $\kappa=0$ and 1 , respectively). 


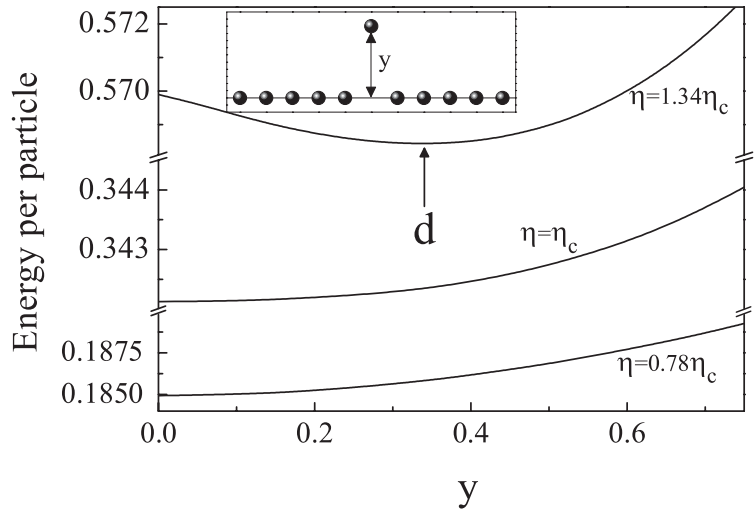

FIG. 6. Potential energy per particle as a function of the position of one of the particles in the confinement direction, before $(\eta=$ $\left.0.78 \eta_{c}\right)$, after $\left(\eta=1.34 \eta_{c}\right)$, and at the transition $\left(\eta=\eta_{c}\right)$ for parabolic confinement. We took the parameters $v=1, \kappa=1$, and $n=1$.

\section{RESULTS AND DISCUSSION}

As has been found in the preceding section, a continuous zigzag transition occurs for parabolic confinement. For the case $\alpha \neq 2$, it is not possible to define a transition between the one- and two-chain configuration because the confinement potential does not contribute to the second-order term of the total potential energy. Additionally, we find that the minimum value of the transverse frequency is purely imaginary [see Eqs. (15a) and (16a)], and this condition implies that, in this case, the transition for the one- to the two-chain configuration is not allowed, which agrees with previous ${ }^{23}$ results. We also performed Monte Carlo simulations and found that, for $\alpha>2$, the one-chain configuration is never formed for any value of the density and the confinement frequency. However, from similar simulations, one can show that, for $\alpha<2$, the one-chain configuration is stable until a critical point, beyond which the configuration is changed to a single chain containing vacancies due to jumps of individual particles away from the chain axis.

\section{A. Transition point for $\alpha \leqslant 2$}

For the case of a power-law interparticle potential $(\kappa=0)$ with parabolic confinement and using dimensionless units, it is possible to find an analytical relationship between the confinement frequency and the linear density as $\eta=v^{-2 /(n+2)}$. For this case, we show in Fig. 4 the behavior of the order parameter as a function of the linear density for different values of $n$. Dashed curves represent the solution from the
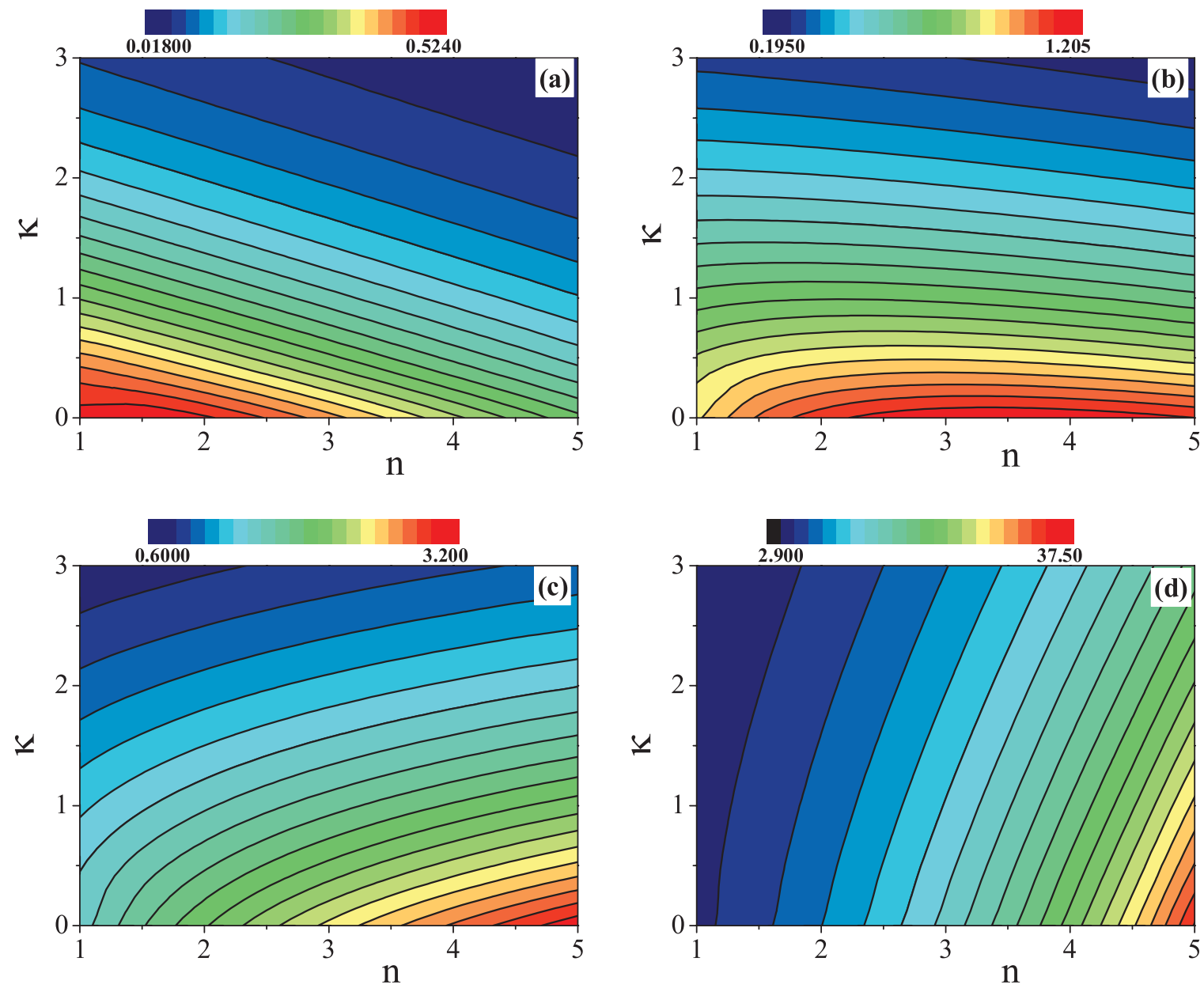

FIG. 7. (Color online) Contour plots of the critical frequency $v_{c}$ as a function of $n$ and $\kappa$ for (a) $\eta=0.50$, (b) $\eta=0.75$, (c) $\eta=1.00$, (d) $\eta=2.00$ in the case of a parabolic confinement potential. 


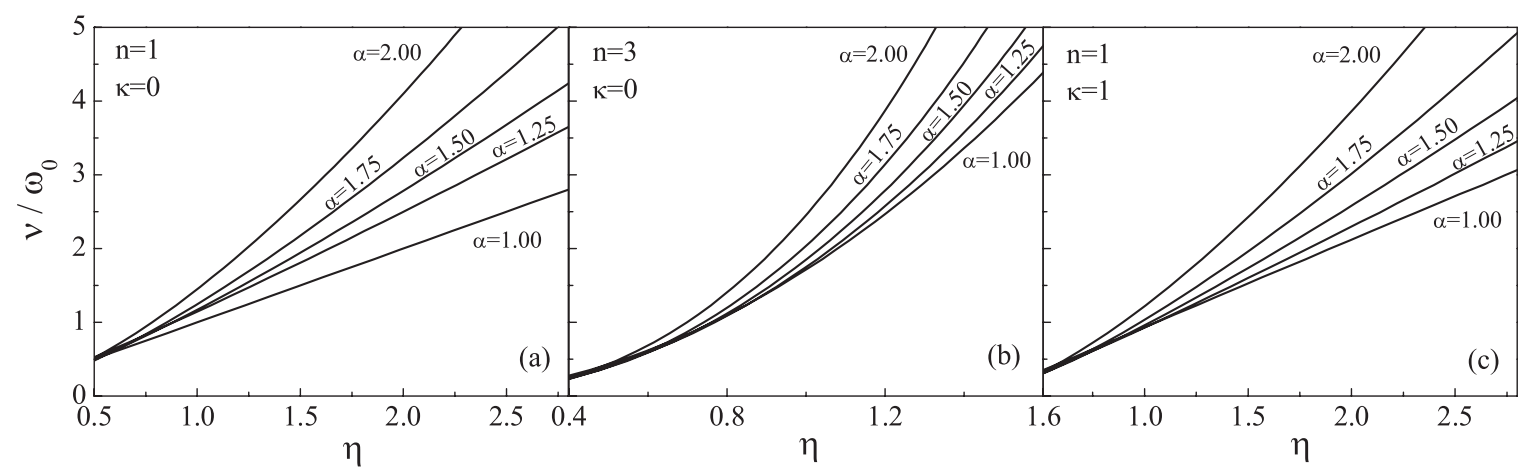

FIG. 8. Critical transition point for the one-chain configuration. Above the curves, the one-chain configuration is the ground state. Results are shown for (a) Coulomb potential, (b) dipole potential, and (c) Yukawa potential for different values of the exponent of the confinement potential $\alpha$.

Landau theory [Eq. (6)], and the full curves are the solution of Eq. (21) and they are compared with the results of a Monte Carlo simulation for $n=1$ and 3 (open circles in Fig. 4). From these results, we notice that there is perfect agreement between our calculation and the exact results obtained from Monte Carlo simulations. In the same context, Fig. 5 shows the variation of the critical density as a function of the exponent $n$ as obtained from Landau theory (solid and dashed curves for $\kappa=0$ and 1 , respectively), and the results from this work (full and open circles for $\kappa=0$ and 1) are shown for different confinement frequencies. Notice that, for $v=1$, this function has a local minimum where the dipole potential exhibits the lowest critical density.

On the other hand, it is also possible to find the value of $d$ numerically by fixing one particle at a distance $y$ from the one-chain axis in the confinement direction and minimize the energy with respect to the position of the other particles. The resulting minimum potential energy of the system is shown in Fig. 6 for a Yukawa interparticle potential with $\kappa=1$ and $v=1$. For $\eta \leqslant \eta_{c}$, the minimum is found at $y=0$, and for $\eta>\eta_{c}$, it continuously shifts to $y \neq 0$, which is typical for a second-order transition.

From Eq. (19), we draw the contour plot of $v_{c}$ as a function of $n$ and $\kappa$ for several values of the density (when making the contour plot, we replaced $n$ by a real number), which are shown in Fig. 7. We observe a strong dependence of the highest value of $v_{c}$ on $\eta$, and therefore the region of frequencies over which the one-chain configuration exists. For low densities $(\eta<1)$, the one-chain organization is dominant for small values of the exponent $n$ and gradually this region is extended to higher values of $n$ with increasing $\eta$. This result shows that, for low densities, the one-dimensional behavior of the system is a better representation for the Coulomb and dipole interparticle potentials. For $\eta \geqslant 1$, the one-dimensional region of frequencies increases with increasing $n$. In all cases, the critical frequency decreases with increasing $\kappa$.

Our previous mean-field theory was derived for modes of the linear chain close to the instability point. Therefore, it is possible to find the critical point for the aforementioned instability from Eq. (27). In Fig. 8, we plot the transition point at which the one-chain structure becomes unstable for $\alpha \leqslant 2$. Above each curve, only $\psi=0$ is a solution of Eq. (27). Only for $\alpha=2$ do the curves correspond to a second-order zigzag transition. Notice that the stability region for the single-chain configuration increases with decreasing $\alpha$.

In Fig. 9(a), we show the dispersion relation for the normal modes in the case of parabolic confinement for different
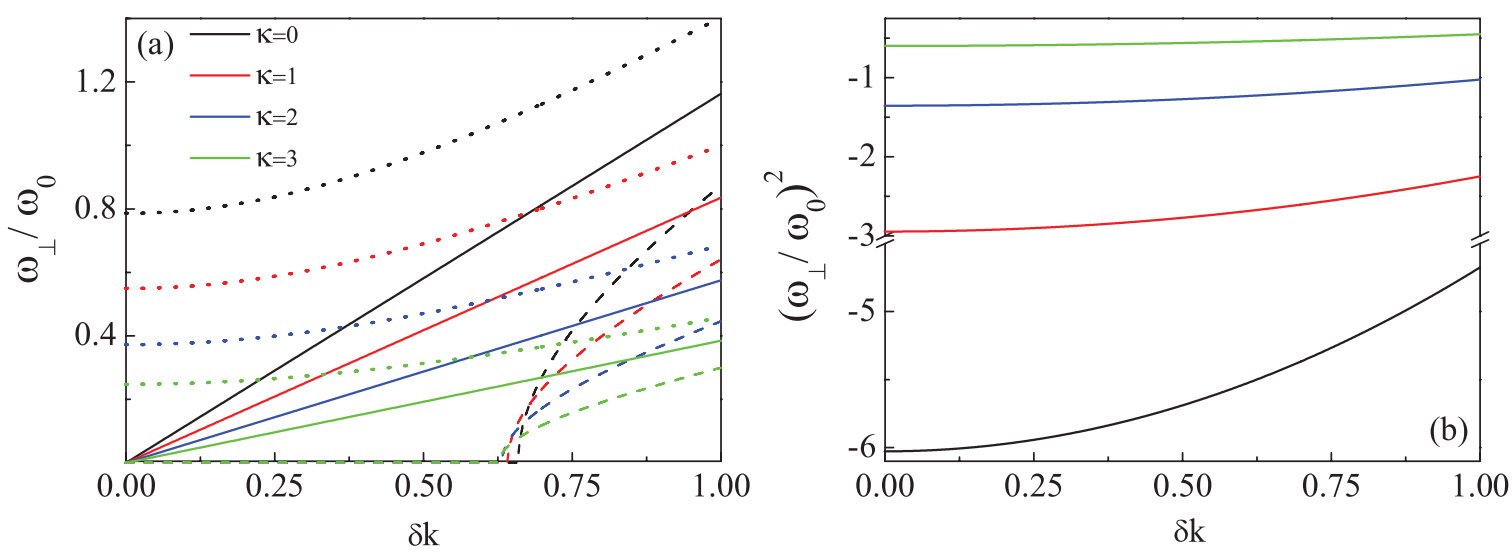

FIG. 9. (Color online) (a) Dispersion relation $\omega_{\perp}$ as a function of $\delta k$ for parabolic confinement (i.e., $\alpha=2$ ) where dashed, solid, and dotted lines represent the results for $v=0.95 v_{c}, v_{c}$, and $1.05 v_{c}$, respectively. (b) Square transverse frequency $\omega_{\perp}^{2}$ as a function of $\delta k$ for $\alpha>2$. We have considered $n=1, \eta=1$ and different values of $\kappa$ as shown in (a). 


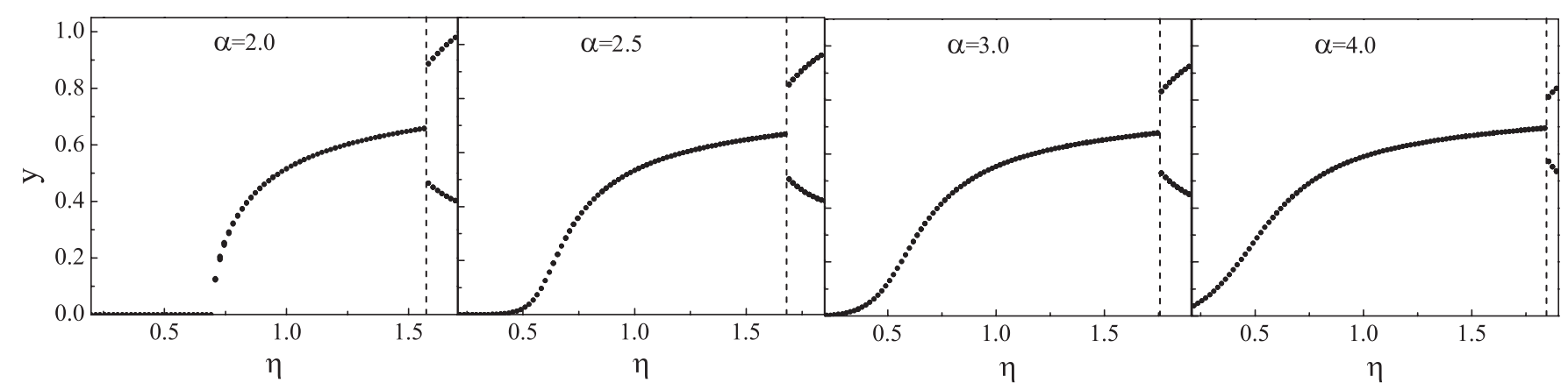

FIG. 10. Distance of the particles from the $y$ axis as a function of the density for different values of $\alpha$ and $v=1$. The interparticle interaction is a dipole potential. The symbols represent the results from Monte Carlo simulations and the dashed vertical lines indicate the transition point from the two- to the four-chain configuration.

values of $\kappa$ in the three cases: linear regime (dotted lines), where the system is stable for any value of the wave vector close to $k_{0}$; the zigzag regime (dashed lines); and in the transition point (solid lines) where the dispersion is linear close to $k_{0}$.

\section{B. Case $\alpha>2$}

In this case, the most simple configuration of the particles is restricted to a two-chain structure; however, from Monte Carlo simulations, we know that there is a transition to a four-chain structure after some value of the linear density. This is shown in Fig. 10, where we plot the distance from the $y=0$ axis of the particles as a function of the density considering a dipole interparticle interaction for different values of $\alpha$. In those figures, the two- to four-chain transition points are marked with a vertical dashed line. In our theoretical model, we have found from Eqs. (15a) and (16a) that $\omega_{\perp}^{2}<0$, as shown in Fig. 9(b), and thus the transverse frequency is imaginary and therefore the one-chain structure is unstable for any value of the density and the confinement strength.

This is illustrated in more detail in Fig. 11, where we plot the distance of the particles from the $x$ axis for different

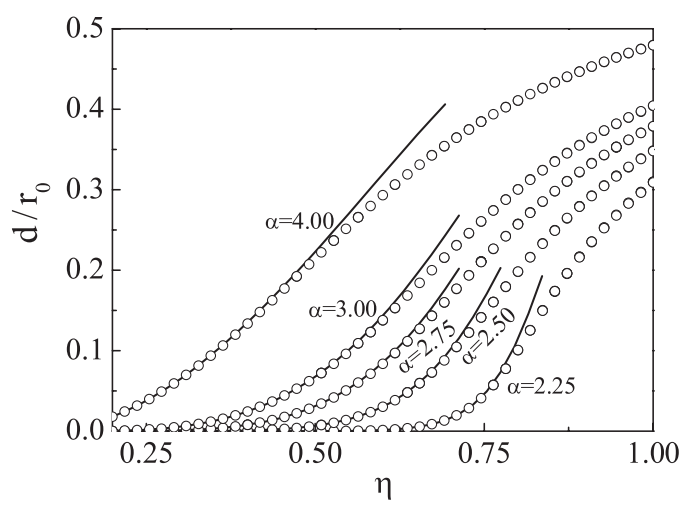

FIG. 11. Order parameter as a function of the linear density in the two-chain region for different values of the exponent of the confinement potential $\alpha$. The solid curves represent the solutions of Eq. (27) and open circles the results of our Monte Carlo simulation. We took the parameters $v=1, \kappa=1$, and $n=1$. values of $\eta$. Note that our mean-field results from Eq. (27) agree with the simulation for small values of $\eta$. Note that, for small values, the confinement potential energy is significantly larger than the interparticle potential energy and, therefore, the fluctuations of the order parameter are smaller. With increasing $\eta$, the interaction between the particles start to dominate and all curves converge to each other (without crossing) for $\eta>1$.

\section{CONCLUSIONS}

In this work, we studied the critical behavior of a system of particles confined in a $2 \mathrm{D}$ channel through a $y^{\alpha}$ potential with different functional forms for the interparticle interaction potential. We derived a Ginzburg-Landau equation for the system and determined the behavior of the system close to the transition point where the single-chain configuration becomes unstable. We determined the order parameter and its dependence on the external confinement and the particle density.

For $\alpha=2$, the critical frequency for the zigzag transition is larger than for smaller values of $\alpha$, which shows that the stability of the linear chain configuration is lower for parabolic confinement. However, for low densities $(\eta<1)$, the one-chain configuration is the most stable state for $\alpha \leqslant 2$.

For $\alpha>2$, the single-chain configuration is unstable for any value of the particle density and the strength of the confinement potential. We found the distance between the two chains as a function of the particle density. With increasing density, a firstorder phase transition is found to the four-chain configuration.

For $\alpha<2$, we found analytically no continuous zigzag configuration irrespective of the interparticle potential. The instability of the single-chain configuration occurs through the expulsion of single particles from the chain to $y \neq 0$ positions.

The instability point for $\alpha=2$ is given by $v_{c}=\eta_{c}^{2 /(n+2)}$, which becomes an almost linear relation, i.e., $v_{c} \sim \eta_{c}$ for $\alpha=$ 1 and $n=1$.

In a future work, we plan to generalize the present analysis to the quantum regime. For the special case of electrons confined by a parabolic potential, i.e., $\alpha=2, n=1$, and $\lambda=\infty$, such an analysis was presented by Meyer et al. ${ }^{30,31}$ Subsequently, the strongly correlated regime, which results in Wigner crystal physics in quantum wires, was addressed in 
Ref. 32. Such a quantum analysis will address the effect of quantum statistics of the particles and the effect of quantum fluctuations on the zigzag transition.

\section{ACKNOWLEDGMENTS}

This work was supported by the Flemish Science Foundation (FWO-Vl). *francois.peeters@ua.ac.be

${ }^{1}$ H. Ikegami, H. Akimoto, and K. Kono, Phys. Rev. Lett. 102, 046807 (2009).

${ }^{2}$ W. M. Itano, J. J. Bollinger, J. N. Tan, B. Jelenkovic, X.-P. Huang, and D. J. Wineland, Science 279, 686 (1998).

${ }^{3}$ I. Waki, S. Kassner, G. Birkl, and H. Walther, Phys. Rev. Lett. 68, 2007 (1992).

${ }^{4}$ D. H. E. Dubin and T. M. O’Neil, Rev. Mod. Phys. 71, 87 (1999).

${ }^{5}$ A. Mortensen, E. Nielsen, T. Matthey, and M. Drewsen, Phys. Rev. Lett. 96, 103001 (2006).

${ }^{6}$ J. I. Cirac and P. Zoller, Phys. Rev. Lett. 74, 4091 (1995).

${ }^{7}$ D. Leibfried, B. DeMarco, V. Meyer, D. Lucas, M. Barret, J. Britton, W. M. Itano, B. Jelenkovic, C. Langer, T. Rosenband, and D. J. Wineland, Nature (London) 422, 412 (2003).

${ }^{8}$ J. M. Taylor and T. Calarco, Phys. Rev. A 78, 062331 (2008).

${ }^{9}$ Y. G. Cornelissens, B. Partoens, and F. M. Peeters, Phys. E (Amsterdam) 8, 314 (2000).

${ }^{10}$ R. W. Hasse and V. V. Avilov, Phys. Rev. A 44, 4506 (1991).

${ }^{11}$ I. V. Schweigert, V. A. Schweigert, and F. M. Peeters, Phys. Rev. B 54, 10827 (1996).

${ }^{12}$ V. A. Schweigert and F. M. Peeters, Phys. Rev. B 51, 7700 (1995).

${ }^{13}$ B. Partoens, V. A. Schweigert, and F. M. Peeters, Phys. Rev. Lett. 79, 3990 (1997).

${ }^{14}$ G. Piacente, I. V. Schweigert, J. J. Betouras, and F. M. Peeters, Phys. Rev. B 69, 045324 (2004).

${ }^{15}$ S. Fishman, G. DeChiara, T. Calarco, and G. Morigi, Phys. Rev. B 77, 064111 (2008).

${ }^{16}$ W. Yang, M. Kong, M. V. Milosevic, Z. Zeng, and F. M. Peeters, Phys. Rev. E 76, 041404 (2007).
${ }^{17}$ A. Valkering, J. Klier, and P. Leiderer, Phys. B (Amsterdam) 284, 172 (2000).

${ }^{18}$ T. Y. M. Chan and S. John, Phys. Rev. A 78, 033812 (2008).

${ }^{19}$ C. Lutz, M. Kollmann, P. Leiderer, and C. Bechinger, J. Phys. Condens. Matter 16, S4075 (2004).

${ }^{20}$ C. Lutz, M. Kollmann, and C. Bechinger, Phys. Rev. Lett. 93, 026001 (2004).

${ }^{21}$ D. Lucena, D. V. Tkachenko, K. Nelissen, V. R. Misko, W. P. Ferreira, G. A. Farias, and F. M. Peeters, e-print arXiV:1010.4540v1.

${ }^{22}$ J. B. Delfau, C. Coste, and M. S. Jean, Phys. Rev. E 84, 011101 (2011).

${ }^{23}$ G. Piacente, G. Q. Hai, and F. M. Peeters, Phys. Rev. B 81, 024108 (2010).

${ }^{24}$ A. del Campo, G. D. Chiara, G. Morigi, M. B. Plenio, and A. Retzker, New J. Phys. 12, 115003 (2010).

${ }^{25}$ T. E. Sheridan and A. L. Magyar, Phys. Plasmas 17, 113703 (2010).

${ }^{26}$ T. L. L. Closson and M. R. Roussel, Can. J. Chem. 87, 1425 (2009).

${ }^{27}$ T. E. Sheridan and K. D. Wells, Phys. Rev. E 81, 016404 (2010).

${ }^{28}$ K. Yosida, Functional Analysis, 3rd ed. (Springer, Berlin, 1971), pp. 153-154.

${ }^{29}$ L. D. Landau and E. M. Lifshitz, Statistical Physics, 3rd ed. (Pergamon, New York, 1986), pp. 179-181.

${ }^{30}$ J. S. Meyer, K. A. Matveev, and A. I. Larkin, Phys. Rev. Lett. 98, 126404 (2007)

${ }^{31}$ T. Meng, M. Dixit, M. Garst, and J. S. Meyer, Phys. Rev. B 83, 125323 (2011)

${ }^{32}$ J. S. Meyer and K. A. Matveev, J. Phys. Condens. Matter 21, 023203 (2009). 